\title{
H3K4 histone methylation in oral squamous cell carcinoma
}

\author{
Marta Mancuso ${ }^{1}$, Danilo Swann Matassa ${ }^{1}$, Mariachiara Conte $^{1}$, Giuseppe Colella ${ }^{2}$, \\ Gina Rana ${ }^{1}$, Laura Fucci ${ }^{1}$ and Marina Piscopo ${ }^{1 凶}$ \\ ${ }^{1}$ Dipartimento di Biologia Strutturale e Funzionale, Università di Napoli Federico II, Napoli, Italy; \\ ${ }^{2}$ Dipartimento di Patologia della Testa e del Collo, Seconda Università di Napoli, Napoli, Italy
}

Received: 19 February, 2009; revised: 23 August, 2009; accepted: 11 September, 2009

available on-line: 14 September, 2009

\begin{abstract}
Methylation of specific lysine residues in histone tails has been proposed to function as a stable epigenetic marker that directs biological functions altering chromatin structure. Recent findings have implicated alteration in heterochromatin formation as a contributing factor in cancer development. In order to verify whether changes in the overall level of $\mathrm{H} 3 \mathrm{~K} 4$ histone methylation could be involved in oral squamous carcinoma, the levels of H3K4me1, me2 and me3 were measured in oral squamous carcinoma, leukoplakias and normal tissues. The levels of H3K4me2 and me3 were significantly different in oral squamous cell carcinoma in comparison with normal tissue: the level of $\mathrm{H} 3 \mathrm{~K} 4 \mathrm{me} 2$ was increased while that of $\mathrm{H} 3 \mathrm{~K} 4 \mathrm{me} 3$ decreased. No significant differences could be found between the two types of tissues in the level of H3K4me1. A similar trend was found in the leukoplakias that appeared more like the pathological than normal tissue. These results support the idea that alteration of chromatin structure could contribute to oncogenic potential.
\end{abstract}

Keywords: H3K4, histone methylation, oral squamous carcinoma

\section{INTRODUCTION}

Evidence accumulated over the past few years suggests that post-translational modifications of histone amino termini constitute a "histone code" that directs a variety of processes involving chromatin (Jenuwein \& Allis, 2001). Histone methylation represents the most recently recognized component of the histone code. Methylation of specific lysine residues in histone tails has been proposed to function as a stable epigenetic marker that directs biological functions ranging from transcriptional regulation to heterochromatin assembly (Völkel \& Angrand, 2007). Methylation of a specific lysine residue (at position 4 and 9 in the amino acid chain) in histone $\mathrm{H} 3$ has been shown to be involved in the regulation of chromatin structure (Lachner \& Jenuwein, 2002).

H3K9 methylation is generally associated with transcriptionally inactive heterochromatin, while K4 methylation is associated with transcriptionally ac- tive euchromatin (Lachner \& Jenuwein, 2002). Lysine methylation on the $\varepsilon$-nitrogen can occur as mono-, di-, or trimethylated forms.

Histone methylation is reversible and dynamically regulated as suggested by the identification of the H3K4-specific histone demethylase LSD1 (Shi et al., 2004) and the H3K36me2-specific histone demethylase JHDM1 (Tsukada et al., 2006). Both LSD1 and JHDM1 demethylate only di- or monomethylated histones while some enzymes of the jumonji class can demethylate also me3 (Völkel \& Angrand, 2007). So far all known lysine histone methyltransferases with the exception of Dot1/DOT $1 \mathrm{~L}$ contain a conserved methyltransferase domain, termed SET (Su(var)3-9, Enhancer-of-zeste, Trithorax) domain, of about 130 amino acids. Recently many connections have been reported between SETdomain proteins that might have a lysine methyltransferase activity and cancer, suggesting that this enzymatic activity is implicated in carcinogenesis.

${ }^{\square}$ Corresponding author: Marina Piscopo, Dipartimento di Biologia Strutturale e Funzionale, Università di Napoli Federico II, via Cinthia, 80126, Napoli, Italy; phone and fax: (39) 081679081; e-mail: piscopo@unina.it

Abbreviations: SET, Su(var)3-9, Enhancer-of-zeste, Trithorax; OSCC, oral squamous cell carcinoma; H3K4, lysine four of histone H3; A.U., arbitrary units; DTT, dithiothreitol; H, oral healthy mucosae; L, oral leukoplakias; C, oral squamous cell carcinomas; PMSF, phenylmethylsulfonyl fluoride. 
Probably these SET-domain proteins will join several other families of chromatin modifying enzymes (acetylases, deacetylases and kinases) that are already implicated in cancer (Kouzarides, 1999). There are examples in which the appearance of a methyl group on either lysines or arginines has been correlated with genes being activated or repressed. One such case is the cyclin E promoter, whose activity is repressed by H3K9 methylation in the G1 phase of the cell cycle (Nielsen et al., 2001).

Oral squamous cell carcinoma (OSCC) is one of the ten most common cancers. It is more common in men and its prevalence increases with age. Similarly, among all potentially malignant lesions and conditions of the oral mucosa, oral leukoplakia (OL) is the most common and extensively studied (Van der Waal et al., 1997; Reibel, 2003). Biological markers that can help to identify the lesions with an aggressive phenotype and worse prognosis need to be studied for both type of oral lesions.

The aim of this work is to characterize the oral squamous carcinoma at a molecular level, measuring the total amount of different forms of H3K4 methylated histone. Our working hypothesis is based on the possibility that levels of mono-, di- and trimethylation of $\mathrm{H} 3 \mathrm{~K} 4$ are different in cancerous oral tissues compared to normal tissues. To this aim we measured the content of K4-methylated $\mathrm{H3}$ histone in healthy, potentially malignant, and cancerous oral tissues in order to evaluate their usefulness as markers of the proliferation status.

\section{MATERIALS AND METHODS}

Acid-soluble protein extraction. Oral tissue $(10-50 \mathrm{mg})$ was washed with PBS $(0.137 \mathrm{M}$ $\mathrm{NaCl}, 1.76 \mathrm{mM} \mathrm{KH} \mathrm{PO}_{4}, 2.7 \mathrm{mM} \mathrm{KCl}, 10.14 \mathrm{mM}$ $\mathrm{Na}_{2} \mathrm{HPO}_{4}$, pH 7.4) and then treated with 7 volumes of lysis buffer $(10 \mathrm{mM}$ Hepes pH 7.9, $10 \mathrm{mM} \mathrm{KCl}$, $1.5 \mathrm{mM} \mathrm{MgCl}$, $0.5 \mathrm{mM}$ DTT, $1.5 \mathrm{mM}$ PMSF). Tissue was manually ground with a sterile pestle. After this treatment $\mathrm{HCl}$ was added to a final concentration of $0.2 \mathrm{M}$ and the samples were kept in ice for $30 \mathrm{~min}$. The samples were centrifuged at 13000 r.p.m. for $10 \mathrm{~min}$ at $4^{\circ} \mathrm{C}$. Protein concentration of the supernatant was determined by BioRad assay (Bradford, 1976) that involves the addition of an acidic dye to protein solution and subsequent measurement at $595 \mathrm{~nm}$ with a spectrophotometer. Protein concentration of the supernatant was verified by Coomassie staining of sodium dodecyl sulphate (SDS)/polyacrylamide gels.

Nine different specimens were analyzed for each: oral healthy mucosae $(\mathrm{H})$, oral leukoplakias (L) and oral squamous cell carcinomas (C). Only histologically confirmed samples were used. An analo- gous procedure was performed to extract acid-soluble proteins from HeLa cells.

Experiments were undertaken with the understanding and written consent of patients and the protocol was approved by a Bioethical Committee.

Western blotting analysis. Acid-soluble proteins $(10 \mu \mathrm{g})$ from oral tissue were resolved by electrophoresis on $12 \%$ SDS/polyacrylamide gels and transferred to nitrocellulose membranes (HybondC, Amersham). Acid-soluble proteins (5 and $10 \mu \mathrm{g}$ ) extracted from HeLa cells were used as a quantitative standard in Western blotting analysis. Polymers of cytochrome $c$ from monomers to heptamers were used as molecular mass standards, as reported in Fig. 1 (Panel A). Three different primary antibodies were used in independent experiments to detect methylated histone $\mathrm{H} 3$ in the protein samples: antimonomethyl Histone H3(K4), anti-dimethyl Histone $\mathrm{H} 3(\mathrm{~K} 4)$, and anti-trimethyl Histone H3(K4), all purchased from Upstate. The anti-monomethyl one was diluted $1: 250$ in $5 \%$ dry milk in TBS (10 mM Tris/ $\mathrm{HCl} \mathrm{pH} 7.5,150 \mathrm{mM} \mathrm{NaCl}$ ) with $1 \% \mathrm{BSA}$ and $0.05 \%$ Tween 20 and incubated for $1 \mathrm{~h}$; the anti-dimethyl antibody was diluted $1: 10000$ in 3\% dry milk in PBS and incubated for $90 \mathrm{~min}$; the anti-trimethyl one was diluted 1:250 in 5\% dry milk in TBS and incubated for $1 \mathrm{~h}$. Incubation was always performed at room temperature. Membranes were then washed two times with water and incubated with peroxidase-conjugated monoclonal antibody anti-rabbit IgG (Sigma) at a dilution of 1:5000. Detection was performed using the ECL plus chemiluminescent kit (Amersham), as described by the manufacturer. Film scanning was done on a Gel DOC 1000 system (Bio$\mathrm{Rad}$ ) and the chemiluminescence was quantified by Molecular Analist 2.1 version software (BioRad). The methylation level of $\mathrm{H} 3$ histone was reported as arbitrary units (A.U.) that represent the ratio between the band intensity in the samples and that in the HeLa cell extract.

As a negative control experiments were repeated skipping the step of membrane incubation with primary antibodies and no bands were revealed (not shown).

\section{RESULTS}

Histone methylation is an important epigenetic mark that allows transcriptional regulation. Particularly, H3 lysine 4 histone methylation is involved in transcriptional activation in several organisms, including humans. In the present work, levels of H3K4 mono-, di- and trimethylation were analysed in oral mucosae of individuals affected by carcinoma or leukoplakia and in clinically healthy individuals as control. Characteristics, risk factors and levels 
Table 1. Characteristics, risk factors and H3K4 mono-, di- and trimethylation levels of specimens analysed in this study.

N.D. means not determined.

Healthy patients $(\mathrm{H})$

\begin{tabular}{|c|c|c|c|c|c|c|c|c|c|c|c|c|}
\hline Individuals & Gender & Age & Smoking & Alcohol & Localization & Diagnosis & Clinical stage & Candida & HPV DNA & MONO & DI & TRI \\
\hline 1 & $M$ & 28 & $20 / d$ & none & gingiva & healthy & - & none & Negative & 51 & 21 & 70 \\
\hline 2 & M & 39 & $10 / d$ & none & gingiva & healthy & - & none & Negative & 45 & 31,5 & 70 \\
\hline 3 & $F$ & 62 & none & $250 \mathrm{cc} / \mathrm{d}$ & gingiva & healthy & - & C. albicans & N.D. & 28 & 37 & 100 \\
\hline 4 & $F$ & 45 & none & none & tongue & healthy & - & none & Positive & 49 & 18 & 66 \\
\hline 5 & M & 58 & ex smoker & none & gingiva & healthy & - & C. albicans & Negative & 36 & 38 & 88 \\
\hline 6 & $F$ & 55 & $15 / d$ & $350 \mathrm{cc} / \mathrm{d}$ & buccal mucosa & healthy & - & none & N.D. & 28 & 9 & 86 \\
\hline 7 & $\mathrm{~F}$ & 37 & none & none & gingiva & healthy & - & none & N.D. & 31 & 12 & 130 \\
\hline 8 & M & 53 & $20 / d$ & none & tongue & healthy & $\cdot$ & none & N.D. & 49 & 39 & 105 \\
\hline 9 & $M$ & 46 & $20 / d$ & none & buccal mucosa & healthy & & none & Negative & 40 & 25 & 122 \\
\hline
\end{tabular}

Patients with oral leukoplakia (L)

\begin{tabular}{|cccccccccccccc}
\hline Individuals & Gender & Age & Smoking & Alcohol & Localization & \multicolumn{2}{c}{ Diagnosis } & Clinical stage & Candida & HPV DNA MONO & DI & TRI \\
\hline 1 & M & 44 & $10 / \mathrm{d}$ & none & buccal mucosa & leukoplakia non homogeneous & - & none & Negative & 83,7 & 93 & 28,9 \\
2 & M & 57 & $20 / \mathrm{d}$ & none & buccal mucosa & leukoplakia & - & C. albicans & Negative & 100 & 100 & 16 \\
3 & F & 46 & $30 / \mathrm{d}$ & none & buccal mucosa & leukoplakia & - & C. albicans & N.D. & 60 & 94 & 12 \\
4 & M & 58 & $40 / d$ & $250 \mathrm{cc} / \mathrm{d}$ & tongue & leukoplakia & - & C. albicans & Negative & 78 & 73 & 14 \\
5 & F & 50 & none & none & gingiva & leukoplakia & - & C. albicans & Negative & 70 & 120 & 16 \\
6 & F & 43 & $30 / d$ & none & buccal mucosa & leukoplakia & - & C. albicans & N.D. & 96 & 60 & 24 \\
7 & M & 83 & none & none & palate & proliferative verrucous leuko & - & C. albicans & Negative & 74 & 71 & 43 \\
8 & F & 61 & $10 / d$ & none & tongue & leukoplakia & - & C. tropicalis & Negative & 87 & 104 & 51 \\
9 & F & 55 & $20 / d$ & none & palate & leukoplakia & - & none & Negative & 105 & 122 & 55 \\
\hline
\end{tabular}

Patients with squamous cell carcinoma (C)

\begin{tabular}{|c|c|c|c|c|c|c|c|c|c|c|c|c|}
\hline Individuals & Gender & Age & Smoking & Alcohol & Localization & Diagnosis & Clinical stage & Candida & HPV DNA & MONO & DI & TRI \\
\hline 1 & $M$ & 67 & none & $250 \mathrm{cc} / \mathrm{di}$ & tongue & SCC & I & C. albicans & Negative & 39,5 & 58,8 & 57 \\
\hline 2 & $M$ & 74 & none & none & tongue & SCC & I & C. famata & Negative & 43 & 65 & 29 \\
\hline 3 & $\mathbf{F}$ & 75 & none & none & buccal mucosa & SCC & IV & C. albicans & Negative & 23 & 74 & 59 \\
\hline 4 & M & 70 & none & $500 \mathrm{cc} / \mathrm{di}$ & tongue & SCC & IV & C. tropicalis & Negative & 40 & 43 & 27 \\
\hline 5 & $\mathbf{F}$ & 80 & none & none & tongue & SCC & III & none & Positive & 40 & 79,4 & 59 \\
\hline 6 & $\mathbf{F}$ & 61 & $30 / d$ & none & floor & SCC & IV & C. cerevisiae & N.D. & 39,8 & 28 & 58 \\
\hline 7 & M & 72 & $20 / d$ & none & lip & $\operatorname{scc}$ & III & C. albicans & Negative & 33 & 38 & 77 \\
\hline 8 & M & 43 & $20 / d$ & $150 \mathrm{cc} / \mathrm{di}$ & buccal mucosa & SCC & III & C. albicans & N.D. & 50 & 69 & 74 \\
\hline 9 & $M$ & 63 & $20 / d$ & $750 \mathrm{cc} / \mathrm{di}$ & floor & SCC & IV & C. kefyr & Negative & 47 & 74 & 74 \\
\hline
\end{tabular}

of H3K4 mono-, di- and tri-methylation of the specimens analysed in this study are reported in Table 1, but it is difficult to correlate the data of methylation with these parameters because of the small number of samples.

In order to measure levels of H3K4 methylation, Western blotting experiments were performed using specific antibodies. It is important to note (Fig. 1, Panel A) that only one band of the expected molecular mass was found in all samples when anti-mono or anti-dimethyl H3K4 antibodies were used. Anti-trimethyl H3K4, instead, revealed one band of a high molecular mass and, in some cases, small amounts of multiple bands with various lower molecular masses. These results suggest that trimethylated H3K4 histone showed a tendency to form polymers/complexes in the experimental conditions used.

Results for three individuals are reported in Fig. 1 (Panel A).

Original data on the other eight healthy individuals, eight patients with leukoplakia and eight patients with carcinoma are reported in Fig. 1 (Pan- el B). The histograms of the average values of mono-, di- and trimethylation levels in carcinomas, leukoplakias and healthy mucosae are reported in Fig. 2.

The methylation levels of H3 histone are reported in arbitrary units (A.U.), comparing the band intensity of the analysed samples with the bands revealed in the histone preparation from HeLa cells on the same films. Carcinomas and leukoplakias show the same behaviour of $\mathrm{H} 3 \mathrm{~K} 4$ methylation in comparison with the healthy oral mucosae, although not the same absolute values. In particular, they both show an increased dimethylation and an even more evident reduced trimethylation level compared to healthy mucosae. On the other hand, only carcinomas showed no significant differences in monomethylation level in comparison to healthy mucosae.

\section{DISCUSSION}

The $\varepsilon$-amine group of certain lysine residues in histones can undergo methylation that can occur 

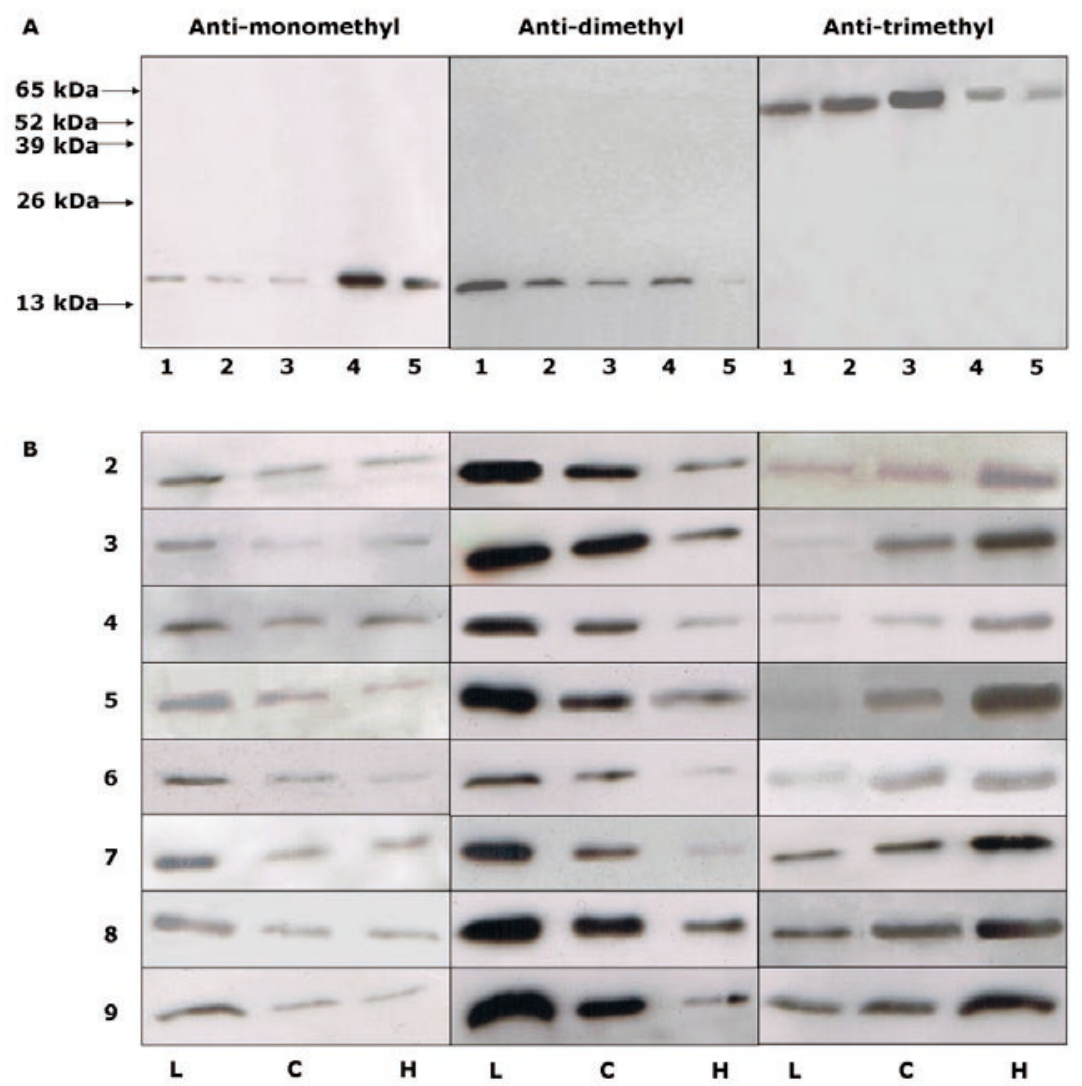

Figure 1. Western blotting of acid-soluble proteins from specimens of individuals 1 (Panel A) and other eight individuals (2-9, Panel B).

Panel A. Lanes 1 from leukoplakias; lanes 2 from carcinomas; lanes 3 from healthy mucosae; lanes 4 and 5 from HeLa cells (10 $\mu \mathrm{g}$ and $5 \mu \mathrm{g}$, respectively). Panel B. leukoplakias (L); carcinomas (C); healthy mucosae $(\mathrm{H})$.

once (mono-methylation) or several times (di- or trimethylation); each level of modification can have different biological effects depending on the residue and context. For example, trimethylation of lysine 4 in histone $\mathrm{H} 3$ (H3K4me3) occurs virtually in all active genes whereas $\mathrm{H} 3 \mathrm{~K} 9 \mathrm{me} 3$ occurs in compact pericentromeric heterochromatin which is transcriptionally inert (Völkel \& Angrand, 2007). Moreover, high levels of H3K4 trimethylation appeared to be associated with the $5^{\prime}$ regions and showed a strong positive correlation with transcription rates, active polymerase II occupancy and histone acetylation (Rutherburg et al., 2007). In contrast, patterns of dimethyl H3K4 differ significantly between yeast and vertebrate chromatin: in Saccharomyces cerevisiae, dimethylated H3K4 appears to spread throughout genes and is associated with a transcriptionally "poised" as well as active state (Pokholok et al., 2005), whereas in vertebrates, the majority of H3K4 dimethylation colocalizes with H3K4 trimethylation in discrete zones (Bernstein et al., 2005). Several interactors of methylated H3K4, including the basal transcription factor TFIID, have recently been identified and it has been demonstrated that they bind to the target via different domains (Rutherburg et al., 2007; Vermeulen et al., 2007).
Methylation of histone lysines is catalyzed by several histone-specific methyltransferases that belong to a novel family whose members share a conserved catalytic motif known as the SET domain; this domain has been demonstrated to play a tumorsuppress function (Kimkc \& Huang, 2003). Some enzymes, such as SET8, possess narrow substrate specificities, while SET7/9 has a broader substrate specificity and can methylate several targets (Völkel \& Angrand, 2007).

Almost all histone lysine methylations are removable. Two classes of lysine demethylases have recently been identified: the LSD1/BHC110 class (which removes H3K4me1 and me2) and the jumonji class (which removes H3K4me2 and me3, H3K9me2 and me3, and H3K36me2 and me3). The LSD1-mediated demethylation reaction was surprisingly specific; i.e., recombinant LSD1 demethylates lysine 4 of histone $\mathrm{H} 3$, but not other methylated arginines or lysines (Bannister \& Kouzarides, 2005). Unlike LSD1, the JmjC domain demethylases do not require protonated nitrogen in the substrate; in this way they are capable of demethylating not only mono- and dimethylated but also trimethylated lysine residues. In this context, it is interesting to note that JHDM1 and JHDM2 both demethylate only mono- and 


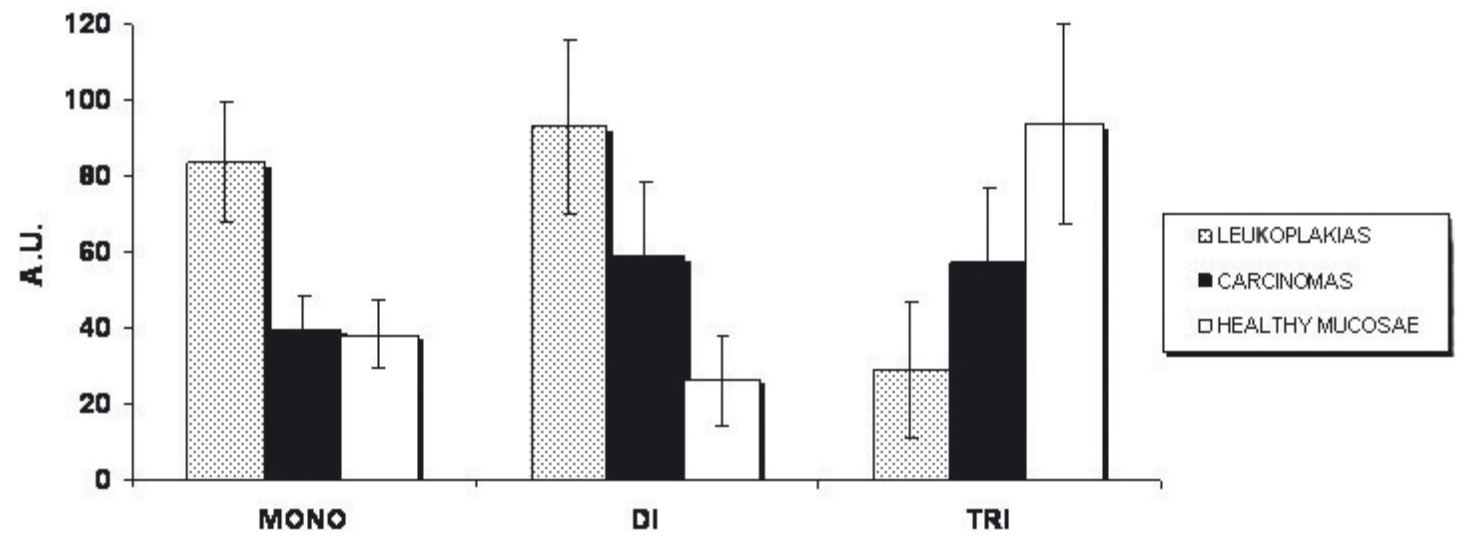

Figure 2. Levels of H3K4 mono-, di- and trimethylation in leukoplakias, carcinomas and healthy mucosae.

Methylation levels are reported in arbitrary units (A.U., see text). The reported results are relative to averages for 9 different specimens ( \pm S.D.).

dimethylated lysine, although they are chemically compatible with the reversal of lysine trimethylation (Völkel \& Angrand, 2007).

Recent findings have implicated alterations in heterochromatin formation as a contributing factor in cancer development (Fraga \& Esteller, 2005). Specifically, a significant reduction of H3K9me3 and H4K20me3 levels, which are hallmarks of heterochromatin, has been correlated with tumorigenesis (Fraga et al., 2005). Therefore, aberrant regulation of the demethylases controlling these methylation marks could contribute to the oncogenic potential. For instance, the JMJD2C/GASC1 gene is amplified in squamous cell carcinoma (Yang et al., 2000). Recently a transcriptional repressor of tumor suppressor genes has been implicated in breast cancer, as a histone demethylase enzyme (PLU-1) that has the ability to reverse the trimethyl H3K4 modification state (Yamane et al., 2007).

Mutations or translocations of histone methyltransferases have been directly linked to prostate, breast and hematopoietic cancers, emphasizing the importance of histone methylation balance in vivo. For instance, the misregulation of MLL (H3K4 methylase), hDOT-1L (H3K79 methylase), and NSD1 (H3K36 methylase) has been linked to human hematopoietic cancers and these data emphasize the need to maintain methylation balance at H3K4, 36 and 79 (Okada et al., 2005).

The data reported in this paper demonstrated that the overall levels of H3K4me2 and me3 are significantly different in oral squamous cell carcinoma in comparison with cells of the healthy tissues; the level of H3K4me2 is increased while that of H3K4me3 decreased. In contrast, no significant differences between the two types of tissues are found in the level of H3K4me1. The same trend in di- and trimethylation was found in the leukoplakias that, in this respect, appeared more similar to the pathological status than to the healthy tissue. These data on the similarity of the leukoplakia with cancer at level of H3K4 methylation support the idea that a high number of leukoplakia are premalignant lesions. It is interesting to understand why leukoplakias show more pronounced changes in methylation than cancer tissues but it is possible that this data are mainly linked to a higher homogeneity of such tissue alterations in comparison with that of carcinomas.

The data on the level of H3K4me2 are in agreement with the results obtained by immunohistochemical analysis on squamous cell cancer and displastic oral lesions (Piyathilake et al., 2005).

In conclusion, on the basis of the reported results, methylation of lysine 4 of histone $\mathrm{H} 3$ is affected in oral squamous cancer.

\section{Acknowledgements}

We thank Professor R. Sartorio for the help in statistical analysis and discussion.

This work was supported by the Ministry of University and Scientific Research (MURST, 2003); Grant No. 2003061804.

\section{REFERENCES}

Bannister AJ, Kouzarides T (2005) Reversing histone methylation. Nature 436: 1103-1106.

Bernstein BE, Kamal M, Lindblad-Toh K, Bekiranov S, Bailey DK, Huebert DJ, McMahon S, Karlsson EK, Kulbokas EJ, Gingeras TR, Schreiber SL, Lander ES (2005) Genomic maps and comparative analysis of histone modifications in human and mouse. Cell 120: 169-181.

Bradford MM (1976) A rapid and sensitive method for the quantitation of microgram quantities of protein utilizing the principle of protein-dye binding. Anal Biochem 72: $248-254$.

Fraga MF, Ballestar E, Villar-Garea A, Boix-Chornet M, Espada J, Schotta G, Bonaldi T, Haydon C, Ropero S, Petrie K, Iyer NG, Pérez-Rosado A, Calvo E, Lopez JA, Cano A, Calasanz MJ, Colomer D, Piris MA, Ahn N, Imhof A, Caldas C, Jenuwein T, Esteller M (2005) Loss of acetylation at Lys16 and trimethylation at Lys20 of 
histone $\mathrm{H} 4$ is a common hallmark of human cancer. Nat Genet 37: 391-400.

Fraga MF, Esteller M (2005) Towards the human cancer epigenome: a first draft of histone modifications. Cell Cycle 4: 1377-1381.

Jenuwein T, Allis CD (2001) Translating the histone code. Science 293: 1074-1080.

Kim KC, Huang S (2003) Histone methyltransferases in tumor suppression. Cancer Biol Ther 2: 491-499.

Kouzarides T (1999) Histone acetylases and deacetylases in cell proliferation. Curr Opin Genet Dev 9: 40-48.

Lachner M, Jenuwein T (2002) The many faces of histone lysine methylation. Curr Opin Cell Biol 14: 286-298.

Nielsen SJ, Scneider R, Bauer UM, Bannister AJ, Morrison A, O'Carroll D, Firestein R, Cleary M, Jenuwein $\mathrm{T}$, Herrera RE, Kouzarides $\mathrm{T}$ (2001) Rb targets histone H3 methylation and HP1 to promoters. Nature 412: 561-565.

Okada Y, Feng Q, Lin Y, Jiang Q, Li Y, Coffield VM, Su L, Xu G, Zhang Y (2005) hDOT1L links histone methylation to leukemogenesis. Cell 121: 167-178.

Piyathilake CJ, Bell WC, Jones JJ, Henao OL, Heimburger DC, Niveleau A, Grizzle WE (2005) Patterns of global DNA and histone methylation appear to be similar in normal, dysplastic and neoplastic oral epithelium. Dis Markers 21: 147-151.

Pokholok DK, Harbison CT, Levine S, Cole M, Hannett NM, Lee TI, Bell GW, Walker K, Rolfe PA, Herbolsheimer E, Zeitlinger J, Lewitter F, Gifford DK, Young RA (2005) Genome-wide map of nucleosome acetylation and methylation in yeast. Cell 122: 517-527.

Reibel J (2003) Prognosis of oral pre-malignant lesions: significance of clinical, histopathological, and molecular biological characteristics. Crit Rev Oral Biol Med 14: 47-62.
Rutherburg AJ, Allis CD, Wysocka J (2007) Methylation of lysine 4 on histone H3: intricacy of writing and reading a single epigenetic mark. Mol Cell 25: 15-30.

Shi Y, Lan F, Matson C, Mulligan P, Whetstine JR, Cole PA, Casero RA, Shi Y (2004) Histone demethylation mediated by the nuclear amine oxidase homolog LSD1. Cell 119: 941-953.

Tsukada Y, Fang J, Erdjument-Bromage H, Warren ME, Borchers CH, Tempst P, Zhang Y (2006) Histone demethylation by a family of JmjC domain-containing proteins. Nature 439: 811-816.

Van der Waal I, Schepman KP, Van der Meij EH, Smeele LE (1997) Oral leukoplakia: a clinicopathological review. Oral Oncol 33: 291-301.

Vermeulen M, Mulder KW, Denissov S, Pijnappel WW, van Schaik FM, Varier RA, Baltissen MP, Stunnenberg HG, Mann M, Timmers HT (2007) Selective anchoring of tfiid to nucleosomes by trimethylation of histone $\mathrm{H} 3$ lysine 4. Cell 131: 58-69.

Völkel P, Angrand PO (2007) The control of histone lysine methylation in epigenetic regulation. Biochimie 89: 120.

Yamane K, Tateishi K, Klose RJ, Fang J, Fabrizio LA, Erdjument-Bromage $\mathrm{H}$, Taylor-Papadimitriou J, Tempst P, Zhang Y (2007) PLU-1 is an H3K4 demethylase involved in transcriptional repression and breast cancer cell proliferation. Mol Cell 23: 801-812.

Yang ZQ, Imoto I, Fukuda Y, Pimkhaokham A, Shimada Y, Imamura M, Sugano S, Nakamura Y, Inazawa J (2000) Identification of a novel gene, GASC1, within an amplicon at 9p23-24 frequently detected in esophageal cancer cell lines. Cancer Res 60: 4735-4739. 